Reprinted with permission. Copyright 2008. The United States

Pharmacopeial Convention. All rights reserved.

\title{
Product Performance Test for Medicated Chewing Gums
}

\author{
Jayachandar Gajendran ${ }^{1,3}$, Johannes Kraemer ${ }^{1,4}$, Stig Randers Knudsen²,4* \\ 'PHAST GmbH, Homburg/Saar, Germany \\ ${ }^{2}$ Fertin Pharma, Vejle, Denmark \\ ${ }^{3}$ Department of Biopharmaceutics and Pharmaceutical Technology, Johannes Gutenberg University, Mainz, Germany \\ ${ }^{4}$ The USP Mucosal Drug Delivery Advisory Committee
}

\section{ABSTRACT}

The nature and type of performance tests used to characterize dosage forms administered to the oral cavity differ significantly and thus demand different release testing apparatus. For many dosage forms given orally but not limited to muco-adhesive buccal tablets, chewable tablets, and sublingual preparations, product performance tests are adapted from existing procedures and are well characterized in the United States Pharmacopeia (USP). Unlike chewable tablets, medicated gums are not supposed to be swallowed and may be removed from the site of application without resort to invasive means. Moreover, medicated gums require the active and continuous masticatory activities for activation and continuation of drug release. Medicated chewing gums are dosage forms given orally for both local and therapeutic effect, and no performance test has been indicated for medicated chewing gums in USP. This Stimuli article outlines the importance and rationale of in vitro drug release testing and the product quality tests used during the development of medicated chewing gums and their quality control for market release.

\section{INTRODUCTION}

M edicated chewing gums are solid or semi-solid pharmaceutical dosage forms and contain one or more active pharmaceutical ingredients (API) and water-soluble or -insoluble excipients blended with a water-insoluble gum base. The drug product is intended to be chewed in the oral cavity for a specific period of time, after which the insoluble gum base is discarded. Medicated chewing gums are defined by the European Pharmacopeia (Ph.Eur.) and the guidelines for pharmaceutical dosage forms issued in 1991 by the Commission of European Communities (CPMP) as "solid, single-dose preparations with a base consisting mainly of gum that are intended to be chewed but not swallowed, providing a slow, steady release of the medicine contained" (1). Drug release from the dosage form is induced by the masticatory process. Two different types of tests are performed to assess the drug product characteristics: product quality and performance tests.

The scope and the differences between the product quality tests and performance tests are clearly defined and have been discussed previously $(2,3)$. Currently, USP contains individual monographs with product quality tests for Nicotine Polacrilex (4) and Nicotine Polacrilex

*Correspondence should be addressed to: Will Brown, Senior Scientist, US Pharmacopeia, 12601 Twinbrook Parkway, Rockville, MD 20852-1790, tel.301.816.8380,e-mailweb@usp.org.
Gum (5). Ph. Eur. has adopted a general monograph on medicated chewing gums $(6,7)$ and a monograph describing the apparatus for dissolution testing of medicated chewing gums $(8-10)$.

\section{MEDICATED CHEWING GUMS}

In Ph. Eur. medicated chewing gums have been separately categorized under solid dosage forms. However, medicated chewing gums also could be defined both as solid and semi-solid preparations based on the art of manufacturing (i.e., using conventional melting procedures) (11) or direct compression of tailored gum-base powder (12). Table 1 shows the product quality tests associated with the chewing gum preparations described in Ph. Eur. in general and specifically with nicotine polacrilex resins and gums in USP. In addition to the product quality tests, additional testing specific to the product may be performed to ensure the final quality of the finished product. This may include, for example, texture analysis, product feel and consistency, evaluation of flavors and sweeteners, tests for coatings, impurities, water content, degradation products, residual solvents, etc. Product performance tests also are included at times by some manufacturers as a quality control tool and are employed during the early phase of product development. USP does not contain a compendial apparatus for performance testing of medicated chewing gums. Thus, in many cases, product performance data were generated by apparatus developed by the drug product manufacturers and are not contained in the public monograph. 
Table 1. Product Quality Tests for Medicated Chewing Gums

\begin{tabular}{lcccc}
\hline $\begin{array}{l}\text { Dosage } \\
\text { Form }\end{array}$ & Nature & $\begin{array}{c}\text { Patient } \\
\text { Activation }\end{array}$ & $\begin{array}{c}\text { Safety Test } \\
\text { Requirement }\end{array}$ & $\begin{array}{c}\text { Product Quality } \\
\text { Tests }\end{array}$ \\
\hline $\begin{array}{l}\text { Medicated } \\
\text { gums }\end{array}$ & $\begin{array}{c}\text { Solid/ } \\
\text { semi-solid }\end{array}$ & Yes & Yes & Assay \\
\cline { 3 - 4 } & & & Identification \\
\cline { 3 - 4 } & & $\begin{array}{c}\text { Uniformity of } \\
\text { dosage units, } \\
\text { content, and mass }\end{array}$ \\
\hline
\end{tabular}

\section{Scientific Rationale for In Vitro Drug Release Determination}

Currently, the USP monograph for nicotine polacrilex gums does not contain a drug release test. Recently, much effort has been spent describing the in vitro release kinetics of special dosage forms, including medicated chewing gums (13-15). Due to the complexity of the release mechanisms involved, researchers proposed minimal requirements for experimental settings with respect to the site of release and absorption. The performance tests, however, must be able to detect the influence of critical manufacturing variables, discriminate between different degrees of product performance, and to some extent, describe the biopharmaceutical quality of finished products.

The scope, purpose, and importance of release tests have been discussed previously (2). Besides the product quality tests, the drug release tests can provide useful information about the characteristics of the product itself, which includes but is not limited to the influence of the composition of the gum and other excipients on drug release, a main tool required primarily during product screening and development, and to some extent the product performance in vivo.

\section{Drug Release Testing Methodology}

Ph. Eur. has adopted an apparatus to determine the release rate from chewing gums formulations (10). The basic principle is a simple masticatory movement employed to simulate the chewing action on a piece of gum placed in a small chewing chamber containing a known volume of buffer solution at a given temperature (10). The drug release rate is influenced by the chewing rate and angle, which provides the necessary shear force to expose new gum surfaces and is a requisite for further drug release.

The mechanism and kinetics of drug release from chewing gums have not yet been completely understood due to the complexity of the formulation itself. The transition from the inactive gum to the active dosage form is influenced by

- mechanical forces

- temperature

- wettability and water permeation.

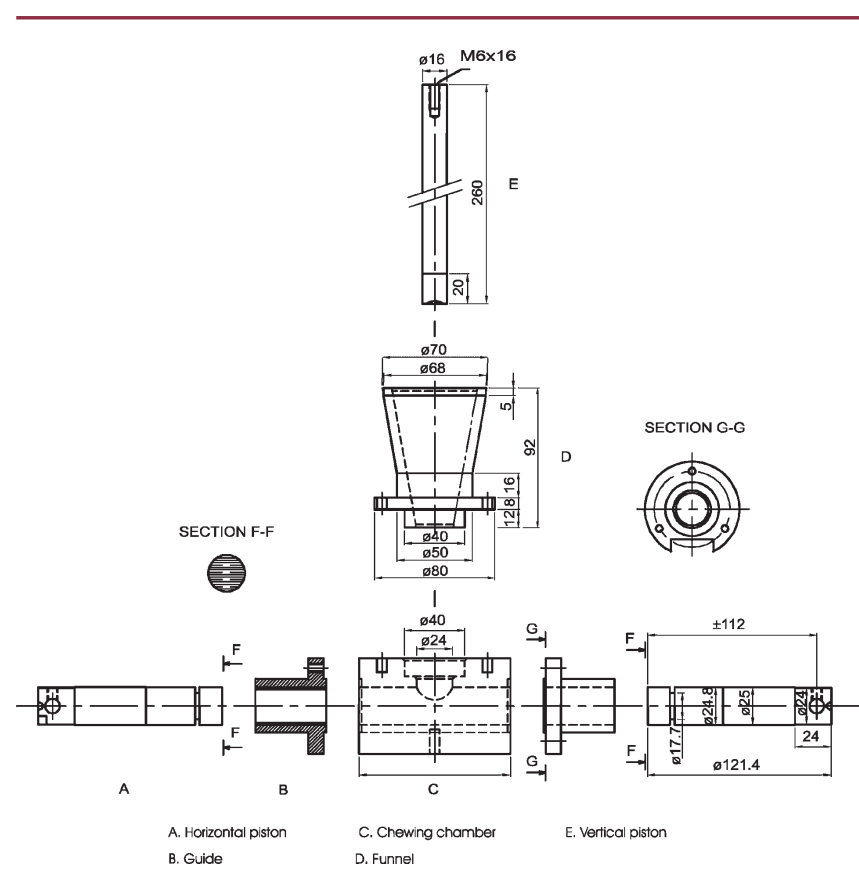

Figure 1. Apparatus for the determination of drug release from medicated chewing gum. Revised with permission from reference (10).

As a general rule, under sink conditions, the rate at which the drug is released is directly proportional to the chewing frequency and aqueous solubility of drug substance and is indirectly proportional to the mass of the gum base.

\section{Apparatus I. Chewing Gum Apparatus, Compendial- Ph. Eur.}

The chewing apparatus for medicated chewing gum was adopted by Ph. Eur. in 2000 (8). Figure 1 shows the construction of the apparatus. The chewing apparatus comprises a chewing chamber, two horizontal pistons, and a third vertical piston (tongue). The vertical piston operates alternatively with the two horizontal pistons and makes sure the gum stays in the right place between chews. If necessary, it is feasible to construct the machine so that at the end of the chew the horizontal pistons rotate around their own axes in opposite directions to each other to obtain maximum chewing. The working procedure of this chewing apparatus is described in Ph.Eur. (8-10). Several studies $(12,15-21)$ have been carried out using the Ph. Eur. apparatus, and the results indicate the methodology is rugged and reproducible.

\section{Apparatus II. Alternative Chewing Gum Apparatus, Noncompendial-Wennergren \\ One of the noncompendial apparatus commercially} available was designed by Wennergren (22). The schematic representation of the Wennergren chewing apparatus is shown in Figure 2. The chewing procedure consists of reciprocations of the lower surface in combination with a shearing (twisting) movement of the upper surface that 


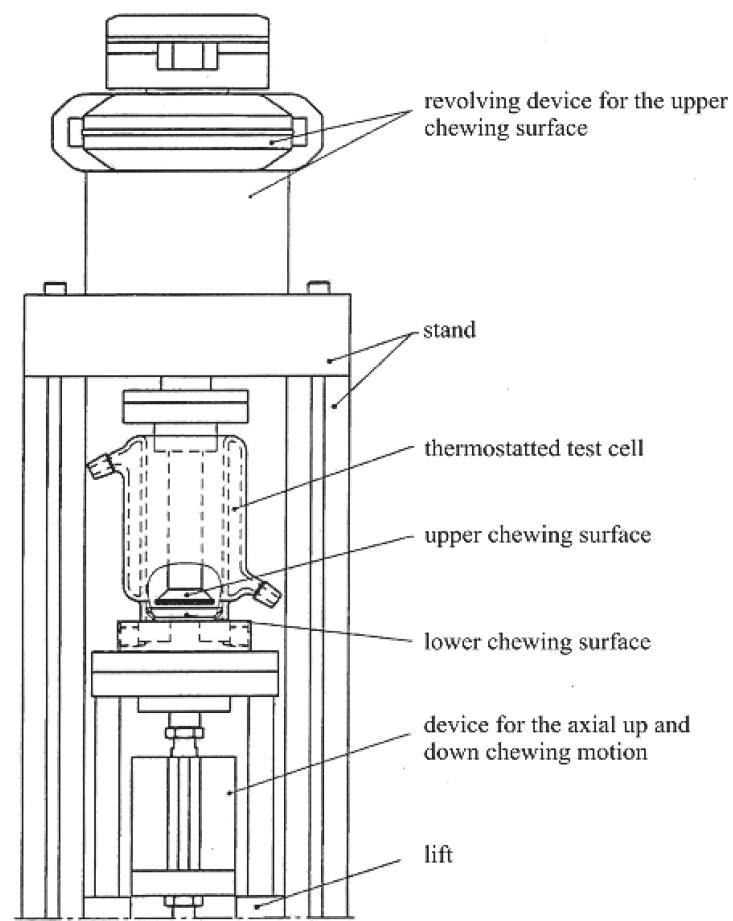

Figure 2. Single-module chewing apparatus from Wennergren (reproduced from publicly available source (22): http://www.fia.se/artikel.pdf).

provides mastication of the chewing gum and at the same time adequate agitation of the test medium. The upper jaw has a flat surface that is parallel to the central part of the lower surface. The small brim of the lower surface is angled upwards (45 degrees) so that the lower surface functions as a small bowl with a flat bottom. This bowl prevents the chewing gum from sliding during mastication.

Investigations of the performance of the chewing apparatus with multiple drug products were published by Wennergren et al. (22). The influences of different operational parameters of the chewing gum apparatus on drug release have been carefully investigated (23).

General Comments about Apparatus I and Apparatus II

Both the apparatus described have been well studied and reported in the literature $(15-18,22-25)$. The results show that the apparatus can provide strong mechanical forces that influence drug release and can prove to be a useful tool for drug release testing of medicated chewing gums both in quality control as well as in product development. To some extent, relevance to in vivo behavior has been demonstrated using both the apparatus $(17,22)$, which suggests their usefulness in the quality control and product development environments.

\section{Product Performance Test for Medicated Chewing Gums}

In vitro drug release testing has become a compendial procedure for many dosage forms. For medicated chewing gums, the performance test ensures the adequate/ required release from a dosage form to provide a local or therapeutic effect. When combined with product quality tests, it serves as a rugged and robust test to ensure the quality of finished products. Both of the apparatus could be successfully employed for performance tests. Before the USP Mucosal Drug Delivery Advisory Committee submits recommendations for medicated chewing gum performance tests to USP, the Advisory Committee suggests that a collaborative study is necessary to evaluate the comparative performance of these apparatus. The study should use drug products from different manufacturers, which would provide a sound scientific rationale to advance an apparatus to compendial status in USP.

\section{SUMMARY}

Further collaborative investigations are required to evaluate and characterize precisely the performance tests for different medicated chewing gum products. These would help identify the appropriate apparatus and its suitability for the performance tests. The USP Mucosal Drug Delivery Advisory Committee is interested in receiving feedback and commentaries on this Stimuli article to expand and refine the scientific knowledge that would ultimately lead to the development of monographs accompanying General Chapters in USP-NF and the development of specifications for medicated chewing gum product performance in individual chapters.

\section{REFERENCES}

1. Commission of the European Communities. CPMP list of allowed terms for the pharmaceutical dosage form, route of administration, container, closure and administration devices; Eurpoean Medicines Agency: Brussels, Belgium, 1991; III/3593/91.

2. Shah, V. P.; Derdzinski, K.; Ewing, G.; et al. A performance test for topical and transdermal dosage Forms. Pharm Forum. 2006, 32 (5), 1586-1589.

3. Shah, V.P.; Ueda, C.T. USP Advisory Panel on the USP Performance Test for Topical and Transdermal Dosage Forms. Pharm Forum. 2006, 32 (5), 1584-1585.

4. United States Pharmacopeia and National Formulary USP 30-NF 25; The United States Pharmacopeial Convention, Inc.: Rockville, MD, 2007; pp 2750-2751.

5. United States Pharmacopeia and National Formulary USP 30-NF 25; The United States Pharmacopeial Convention, Inc.: Rockville, MD, 2007; p 2751.

6. General Monograph on Dosage Forms: Chewing gums, Medicated. In European Pharmacopoeia, 5th ed.; European Directorate for the Quality of Medicines, Council of Europe: Strasbourg, France, 2005; p 601.

7. General Monograph on Dosage Forms. Chewing gums, Medicated. In European Pharmacopoeia, 6th ed.; European Directorate for the Quality of Medicines, Council of Europe: Strasbourg, France, 2008; p 719.

8. Chewing gum, Medicated Release from, General Chapter 2.9.25. In European Pharmacopoeia, 3rd ed.; 
European Directorate for the Quality of Medicines, Council of Europe: Strasbourg, France, 2000; Suppl., p 104.

9. Dissolution Test for Medicated Chewing Gums, General Monograph 2.9.25. In European Pharmacopoeia, 5th ed.; European Directorate for the Quality of Medicines, Council of Europe: Strasbourg, France, 2005; Suppl. 5.2, pp 3116-3117.

10. Dissolution Test for Medicated Chewing Gums, General Monograph 2.9.25. In European Pharmacopoeia, 6th ed.; European Directorate for the Quality of Medicines, Council of Europe: Strasbourg, France, 2008; pp 304-306.

11. Rassing, M. R. Chewing gum as a drug delivery system. Adv. Drug Deliv. Rev. 1994, 13, 89-121.

12. Morjaria, Y.; Irwin, W. J.; Barnett, P. X.; Chan, R. S.; Conway, B. R. In vitro release of nicotine from chewing gum formulations. Dissolution Technol. 2004, 11 (2), 12-15.

13. Siewert, M.; Dressman, J. B.; Brown, C.; Shah, V.P. FIP/ AAPS guidelines for dissolution/in vitro release testing of novel/ special dosage forms. Dissolution Technol. 2003, 2, 6-15.

14. Möller, H.; Shah, V.; Brown, C. Dissolution testing of special dosage forms. Dissolution Technol. 1999, 6 (4), 18-20.

15. Yang, X.; Wang, G.; Zhang, X. Release kinetics of catechins from chewing gum.J.Pharm.Sci. 2004, 93 (2), 293-299.

16. Jensen, E.; Lokind, K. B.; Pedersen, M.; Rassing, M. R. Chewing gum as a drug delivery system-influence of additives upon the rate of drug release of metronidazole and propranolol hydrochloride from chewing gum. Farm. Sci. Ed. 1988, 16, 94-97.

17. Christrup, L. L.; Moeller, N. Chewing gum as a drug delivery system I. in vitro simulation of human mastication and influence of formulation upon the release of a water- soluble drug. Arch. Pharm. Chem. Sci. Ed. 1986, 14, 30-36.

18. Christrup, L. L.; Rassing, M. R. Chewing gum as a drug delivery system: influence of the formulation upon the rate of release of salicylamide. Farm. Sci. Ed. 1988, 16, $1-5$.

19. Faraj, J. A.; Dorati, R.; Schoubben, A.; et al. Development of a peptide-containing chewing gum as a sustained release antiplaque antimicrobial delivery system. AAPS PharmSciTech. 2007, 8 (1), 26.

20. Pedersen, M.; Rassing, M. R. Miconazole and miconazolenitrate chewing gum as drug delivery systems:a practical approach of solid dispersion technique. Drug Dev. Ind. Pharm. 1990, 16 (1), 55-74.
21. Pedersen, M.; Rassing, M. R. Miconazole chewing gum as a drug delivery system test of release promoting additives. Drug Dev. Ind. Pharm. 1991, 17 (3), 411-420.

22. Kvist, C.; Andersson, S. B.; Fors, S.; Wennergren, B.; Berglund, J. Apparatus for studying in vitro drug release from medicated chewing gums. Int. J. Pharm. 1999, 189 (1), 57-65.

23. Kvist, L. C.; Andersson, S. B.; Berglund, J.; Wennergren, B.; Fors, S. M. Equipment for drug release testing of medicated chewing gums. J. Pharm. Biomed. Anal. 2000, 22 (3), 405-411.

24. Andersen, T.; Gram-Hansen, M.; Pedersen, M.; Rassing, M. R. Chewing gum as a drug delivery system for nystatin-influence of solubilizing agents upon the release of water insoluble drugs. Drug Dev. Ind. Pharm. 1990, 16 (13), 1985-1994.

25. Jensen, L. N.; Christrup, L. L.; Menger, N.; Bundgaard, H. Chewing gum and lozenges as delivery systems for noscapine. Acta Pharm. Nord. 1991, 3 (4), 219-222.

\section{Dissolution Solutions Network}

The number one independent Pharma-Lab Blog site on the web with cutting edge information on USP Dissolution testing best suited for your marketing needs.

- Periodic commentaries by pharmaceutical scientists

- The most used collection of links in the dissolution Industry

- Trusted independent reviews

- Breaking pharmaceutical news

- 10 years of history on the internet

For a limited time only, the Dissolution Solutions Network is opening up advertising space at discount prices. Visit the Dissolution Solutions network. Stay informed, stay in touch, and stay current. www.dissolutionsolutions.net 